\title{
Deferoxamine inhibits TRAIL-mediated apoptosis via regulation of autophagy in human colon cancer cells
}

\author{
JI-HONG MOON, JAE-KYO JEONG and SANG-YOUEL PARK \\ Biosafety Research Institute, College of Veterinary Medicine, Chonbuk National University, \\ Jeonju, Jeonbuk 561-756, Republic of Korea
}

Received September 11, 2014; Accepted November 28, 2014

DOI: $10.3892 /$ or.2014.3676

\begin{abstract}
Deferoxamine (DFO), an iron chelator, has numerous clinical applications for patients presenting with iron overload in regards to the improvement in the quality of life and overall survival. In addition, experimental iron chelators have demonstrated potent anticancer properties. The present study investigated the effects of DFO on TNF-related apoptosisinducing ligand (TRAIL)-induced apoptosis in colon cancer cells and and the mechanism involved. The experimental results showed that DFO treatment inhibited TRAILmediated cancer cell apoptosis by increasing Akt activation and decreasing caspase activation in human colon cancer cells. Furthermore, DFO treatment induced autophagy flux, and chloroquine, an autophagy inhibitor, blocked DFO-mediated inhibition of TRAIL-induced apoptosis. The present study demonstrated that DFO inhibited TRAIL-mediated tumor cell death via the autophagy pathway, and the results suggest that potent anticancer agent, DFO, can be an inhibitor against antitumor therapy including TRAIL protein.
\end{abstract}

\section{Introduction}

Apoptosis is a common morphological form of programmed cell death that plays a critical role during development and homeostasis, and in many diseases including cancer, acquired immunodeficiency syndrome and neurodegenerative disorders (1). This type of apoptosis called programmed cell death type I can be triggered in tumor cells by anticancer agents $(2,3)$. Tumor necrosis factor (TNF)-related apoptosisinducing ligand (TRAIL) was first identified as a member of the TNF superfamily and can selectively induce apoptosis in tumorigenic or transformed cells, yet not in normal cells,

Correspondence to: Professor Sang-Youel Park, Biosafety Research Institute, College of Veterinary Medicine, Chonbuk National University, Jeonju, Jeonbuk 561-756, Republic of Korea

E-mail: sypark@chonbuk.ac.kr

Key words: deferoxamine, TRAIL, autophagy, colon cancer highlighting its potential therapeutic application in cancer treatment (4).

Iron $(\mathrm{Fe})$ is a useful target as it plays a critical role in proliferation involved in numerous metabolic pathways such as ATP generation, oxygen transport and DNA synthesis (5). A few pathways have been proposed in regards to iron-related carcinogenesis. Studies indicate that iron-induced oxidative stress may cause DNA, protein and organelle damage, through production of hydroxyl radicals and hydrogen peroxide via Haber-Weiss and Fenton-type reactions (6,7). Some studies suggest that dietary Fe restriction using iron chelators has been shown to decrease tumor proliferation in a number of in vitro and in vivo studies (8-10). Hershko reported that in preliminary studies, deferoxamine (DFO), an iron chelators, in combination with multidrug chemotherapy was effective in controlling several tumors (11).

DFO was developed more than 40 years ago and takes an active part as an iron chelator in the improvement of the quality of life and overall survival of patients presenting with iron overload (12). DFO binds iron tightly, and the iron-DFO complex is excreted in both urine and stool. DFO has been shown to be effective as an antitumor drug through several signaling pathways in tumor cells $(9,10)$. DFO is well known to be used to induce hypoxia-inducible factor- $1 \alpha(\mathrm{HIF}-1 \alpha)$ as it mimics the hypoxic effects at $21 \% \mathrm{O}_{2}(13)$. HIF- $1 \alpha$ is a transcriptional factor composed of $\alpha$ - and $\beta$-subunits and is a key regulator of metabolism to hypoxia (14). HIF-1 is involved in critical aspects of cancer biology such as angiogenesis, cell survival and invasion and intratumoral hypoxia, and genetic alterations can lead to HIF-1 $\alpha$ overexpression, which has been related to increased patient mortality in several types of cancer (15).

Autophagy, 'the eating of self', was first coined by Deter and De Duve several decades ago and is mainly based on the observed degradation response to starvation or stress whereby mitochondria and cellular elements are digested by lysosomes (16). Autophagy, a common morphological feature in dying cells, appears to be one of the major functions to keep cells alive under stressful conditions (17). In cancer cells, autophagy can promote apoptosis in some cases $(18,19)$. However, autophagy has a more crucial role in sustaining cell viability with defects in apoptosis (19-21). Hu et al suggested that hypoxia-induced autophagy promotes tumor cell survival (22). In the present study, we demonstrated that DFO 
inhibits TRAIL-induced apoptosis via regulation of autophagy in colon cancer cells.

\section{Materials and methods}

Cell culture. The human colon cancer cell line HCT116 was obtained from the American Type Culture Collection (ATCC; Manassas, VA, USA). Cells were cultured in RPMI-1640 medium supplemented with $10 \%$ fetal bovine serum (both from Invitrogen-Gibco, Carlsbad, CA, USA), $100 \mathrm{U} / \mathrm{ml}$ penicillin, and $0.1 \mathrm{mg} / \mathrm{ml}$ gentamycin in a humidified incubator maintained at $37^{\circ} \mathrm{C}$ and $5 \% \mathrm{CO}_{2}$. Cells were treated for $24 \mathrm{~h}$ with DFO and then exposed for $6 \mathrm{~h}$ to $200 \mathrm{ng} / \mathrm{ml}$ TRAIL with or without the autophagy inhibitor chloroquine $(10 \mu \mathrm{M})$ (both from Sigma-Aldrich, St. Louis, MO, USA).

Crystal violet assay. Cell morphology was assessed microscopically (inverted microscope, Nikon), and cell viability was determined by crystal violet staining, as previously described (23). Briefly, cells were stained for $10 \mathrm{~min}$ at room temperature with crystal violet solution $(0.5 \%$ crystal violet in $30 \%$ ethanol and $3 \%$ formaldehyde), washed five times with water, and then dried. After that, the cells were lysed with $1 \%$ sodium dodecyl sulphate (SDS), and the absorbance was measured at $550 \mathrm{~nm}$. Cell viability was calculated from the relative dye intensity of the samples compared to the controls.

Trypan blue exclusion assay. The number of viable cells was determined by trypan blue dye exclusion (Sigma-Aldrich) using a hemocytometer. The result was expressed as a percentage relative to the vehicle-treated controls.

Western blot analysis. HCT116 cells were lysed in lysis buffer [25 mM 4-(2-hydroxyethyl)-1-piperazineethanesulfonic acid (HEPES), pH 7.4, $100 \mathrm{mM} \mathrm{NaCl}, 1 \mathrm{mM}$ ethylene diamine tetraacetic acid (EDTA), $5 \mathrm{mM} \mathrm{MgCl}_{2}, 0.1 \mathrm{mM}$ dithiothreitol (DTT) and a protease inhibitor mixture], and whole cell proteins were electrophoretically resolved on a $10-15 \%$ sodium dodecyl sulfate polyacrylamide gel and transferred to a nitrocellulose membrane. Immunoreactivity was detected through sequential incubation with primary antibodies, horseradish peroxidase-conjugated secondary antibodies, and enhanced chemiluminescence reagents (Westsave Gold Detection kit; AbFrontier Inc.). The primary antibodies used for immunoblotting were anti-human HIF-1 $\alpha$ (BD Biosciences), anti-LC3B (Cell Signaling Technology), anti-P62 (Millipore Corporation), anti-phospho-AKT (Epitomics, Burlingame, CA, USA) and anti- $\beta$-actin (Sigma-Aldrich). Images were examined using a Fusion FX7 imaging system (Vilber Lourmat, ZI Sud Torcy, France). The densitometry of the signal bands was analyzed using Bio-1D software (Vilber Lourmat, Marne la Vallée, France).

Statistical analysis. The unpaired t-test or Welch's correction was used for comparison between the two groups. For multiple comparison, the one-way ANOVA followed by the Tukey-Kramer test was used. All statistical analysis was performed using GraphPad Prism software. Results were considered significant for values $\mathrm{p}<0.05, \mathrm{p}<0.01$ or $\mathrm{p}<0.001$.

\section{Results}

DFO inhibits TRAIL-induced cell death in colon cancer cells. We examined whether DFO promotes or inhibits TRAIL-induced cell death and whether this effect is associated with induction of autophagy. We investigated the influence of DFO on TRAIL-mediated cytotoxicity in HCT116 colon cancer cells by images and crystal violet assay. HCT116 cells were exposed to DFO with or without TRAIL. The cell viability of the TRAIL-treated cells was decreased more than half according to images captured by light microscopy and crystal violet assay. The cell viability of the cells treated with DFO only was comparable to that of the untreated controls. These results revealed that DFO treatment inhibited TRAIL-induced cytotoxicity in HCT116 colon cancer cells (Fig. 1A-C). Trypan blue exclusion assay was implemented for cell viability (Fig. 1D).

If cell death is induced by cytotoxicity in cells, then cells cannot generate exocytosis. Thus, trypan blue particles can flow in cells easily. For this reason, trypan blue dyed cells represent dead cells. These comprehensive results exhibited that DFO treatment inhibited cell death induced by TRAIL treatment dose-dependently. These results indicate that DFO was effective in preventing TRAIL-induced cell death in HCT116 colon cancer cells.

DFO mediates HIF-l $\alpha$ stabilization and Akt activation. We identified the proliferative and protective effects of DFO on cancer cells. We hypothesized that DFO would play a role of an HIF-1 $\alpha$ inducer not an iron chelator, since HIF-1 $\alpha$ is involved in critical aspects of cancer biology such as angiogenesis and cell survival (15). We investigated whether DFO induces HIF-1 $\alpha$ stabilization by western blot analysis. As shown in Fig. 2A and $\mathrm{B}$, the level of HIF-1 $\alpha$ was increased in the DFO-treated group in a dose-dependent manner compared with the control group as detected by western blot analysis and densitometry. These results suggest that DFO leads to stabilization of HIF-1 $\alpha$. We investigated whether DFO affects survival and death signals. Akt affects cell survival and metastasis in many types of cells including colon cancer cells. Akt acts as a key signal that is associated with oncogenic receptors to many essential prosurvival cellular functions in human cancer (24). Caspase-3 plays a key role in regulating programmed cell death or apoptosis, a normal process required for maintenance of the regulation of physiological functions (25). Our data showed that DFO treatment recovered Akt activation from reduction by TRAIL treatment (Fig. 2A and C) and decreased the caspase-3 cleavage induced by TRAIL (Fig. 2A and D). Thus, these results suggest that DFO confers a protective effect from TRAIL in colon cancer cells.

DFO induces autophagic flux. LC3 protein is considered to be an autophagy marker and is localized and aggregated on autophagosomes. LC3 transforms from LC3-I to LC3-II during autophagosome formation (26). We examined whether DFO induces autophagy by assessing LC 3 transformation. As shown in Fig. 3, the level of LC3-II was increased and P62 expression was decreased in the DFO-treated group in a dose-dependent manner compared with the control group as detected by western blot analysis and densitometry (Fig. 3A and B). P62 
A

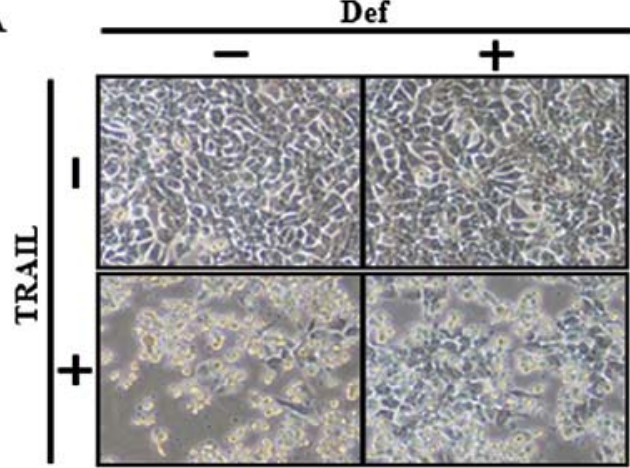

C

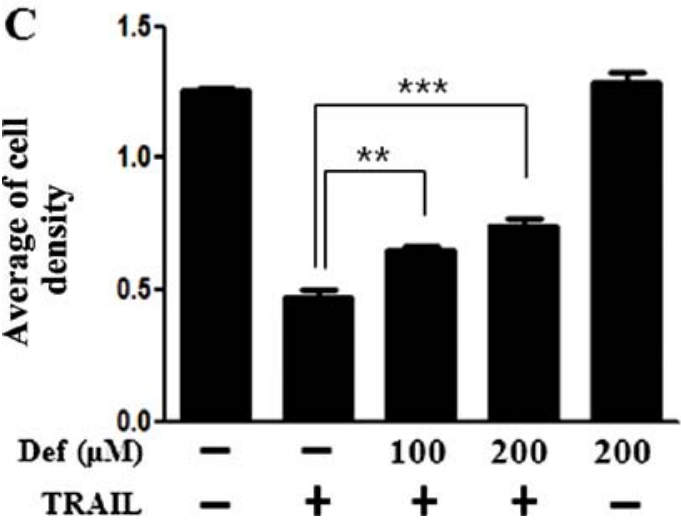

B

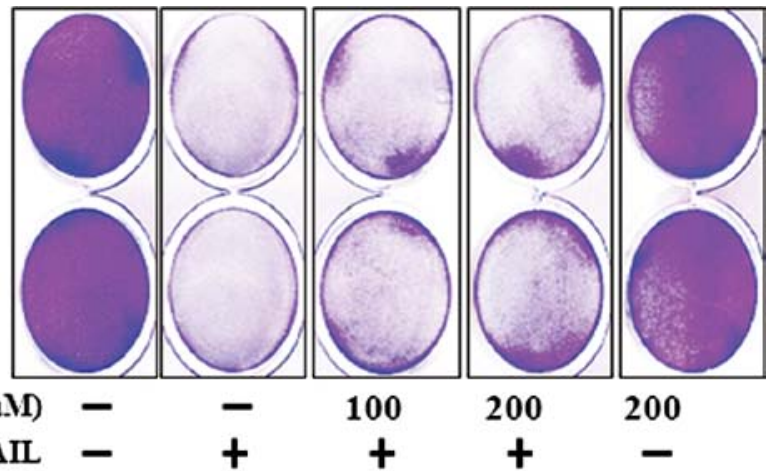

D

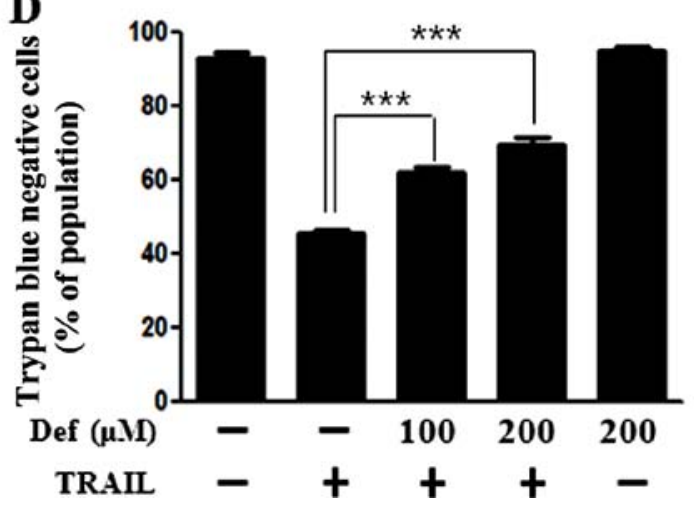

Figure 1. Deferoxamine inhibits TRAIL-induced cell death in colon cancer cells. (A) HCT116 colon cancer cells were pretreated with deferoxamine (Def) $(24 \mathrm{~h}$ ) in a dose-dependent manner, and then exposed to $200 \mathrm{ng} / \mathrm{ml}$ TRAIL for $6 \mathrm{~h}$. Cell viability was measured by imaging with a light microscope (magnification, x100). (B) Cell viability was measured by crystal violet assay. The bar graphs indicate (C) the average density of the crystal violet dyed cells and (D) cell viability as measured by trypan blue dye exclusion assay. ${ }^{*} \mathrm{p}<0.05,{ }^{* *} \mathrm{p}<0.01,{ }^{* * * *} \mathrm{p}<0.001$; significant differences between the control and each treatment group. TRAIL, tumor necrosis factor (TNF)-related apoptosis-inducing ligand.
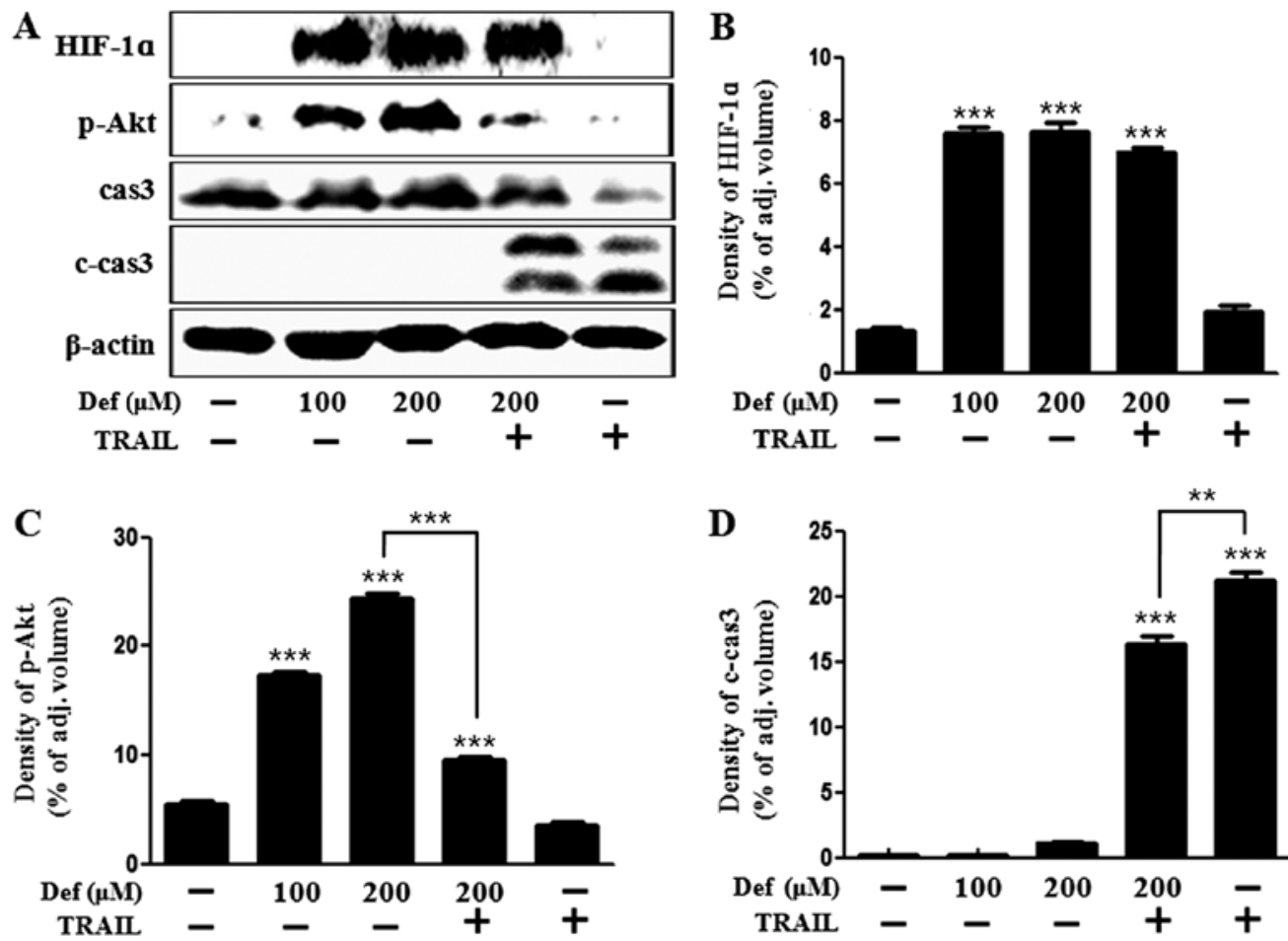

Figure 2. Deferoxamine mediates HIF-1 $\alpha$ stabilization and Akt activation. (A) HCT116 cells were treated with deferoxamine (Def) in a dose-dependent manner. The treated cells were assessed for HIF-1 $\alpha$ and phosphorylation of Akt and caspase 3 cleavage by western blot analysis. Results were normalized to $\beta$-actin. The bar graphs indicate the averages of (B) HIF-1 $\alpha$ stabilization, (C) phosphorylated-Akt and (D) cleaved-caspase 3 ratio. ${ }^{*} \mathrm{p}<0.05,{ }^{* *} \mathrm{p}<0.01$, ${ }^{* * * *} \mathrm{p}<0.001$; significant differences between the control and each treatment group. TRAIL, tumor necrosis factor (TNF)-related apoptosis-inducing ligand; HIF-1 $\alpha$, hypoxia-inducible factor $1 \alpha$; p-Akt, phosphorylation of Akt; cas3, caspase 3; c-cas3, cleaved caspase 3; adj. volume, adjustment of volume (band volume minus background volume). 
$\mathbf{A}$

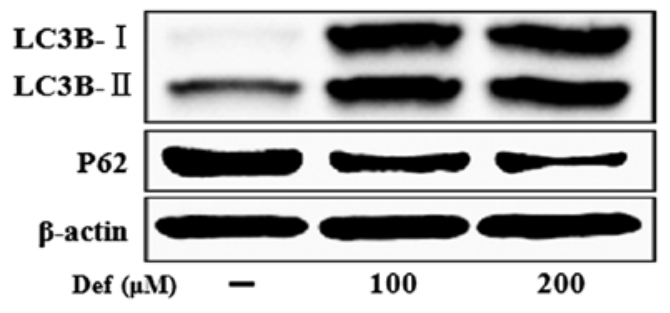

C

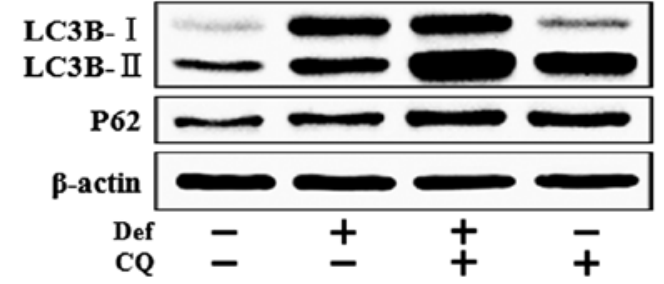

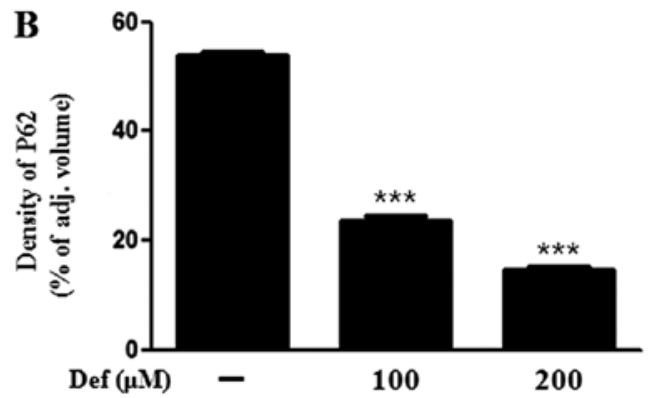

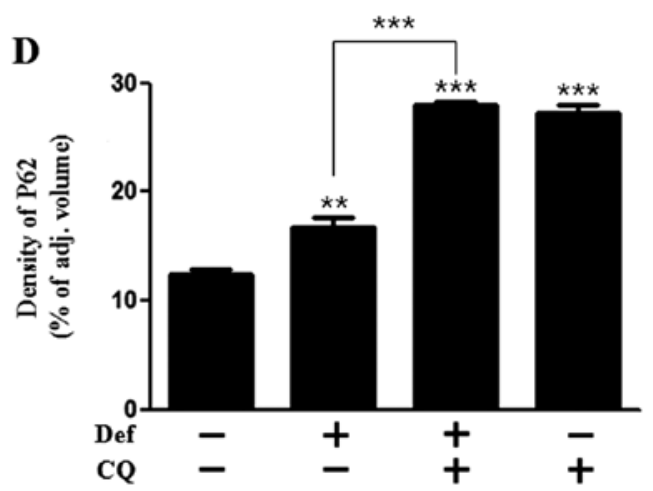

Figure 3. Deferoxamine regulates autophagy. (A) HCT116 colon cancer cells were treated with deferoxamine (Def) (24 h) in a dose-dependent manner. The treated cells were assessed for LC3B production by western blot analysis. Results were normalized to $\beta$-actin. (B) The bar graph indicates the averages of the P62 expression ratio. (C) HCT116 colon cancer cells were pretreated with autophagy inhibitor (chloroquine; CQ) (1 h) and then exposed to deferoxamine $(200 \mu \mathrm{M})$ for $24 \mathrm{~h}$. (D) The bar graph indicates the averages of the P62 expression ratio. ${ }^{*} \mathrm{p}<0.05,{ }^{* *} \mathrm{p}<0.01,{ }^{* * * *} \mathrm{p}<0.001 ;$ significant differences between the control and each treatment group. TRAIL, tumor necrosis factor (TNF)-related apoptosis-inducing ligand; adj. volume, adjustment of volume (band volume minus background volume).

is considered as a crucial mediator for target protein to the autophagy system in removal of aggregated proteins. P62 is degraded by itself during autophagy (27). We used the autophagy inhibitor, chloroquine (CQ), that is widely used to inhibit the maturation of autophagosomes into degradative autolysosomes $(18,28)$. We identified that DFO-induced upregulation of LC3-II was increased by CQ treatment since CQ inhibited the fusion of autophagosomes and autolysosomes (Fig. 3C). For this reason, P62 was increased by CQ treatment (Fig. 3C and D).

Autophagy inhibitor blocks DFO-mediated inhibition of TRAIL-induced apoptosis. We analyzed the cell viability using CQ to investigate the effect of DFO on the activation of autophagy. We identified that the protective effect of DFO was reversed by $\mathrm{CQ}$ in regards to cell viability by crystal violet assay and graphical analysis (Fig. 4A and B). Photographed images and trypan blue exclusion assay were implemented for cell viability. Inhibition of cell death by DFO treatment was reversed by CQ pre-treatment (Fig. 4C and D). These results strongly indicate that DFO treatment inhibits TRAILmediated cytotoxicity through induction of autophagy.

\section{Discussion}

Deferoxamine (DFO), an iron chelator, has been reported to induce hypoxia and HIF-1 $\alpha$ expression (29). HIF-1 $\alpha$ is a crucial mediator of the physiological response to hypoxia, and its dysfunction promotes cancer angiogenesis and metastasis (30).
We identified that DFO induced HIF-1 $\alpha$ stabilization. Several studies indicate that HIF-1 $\alpha$ induces autophagy in the cellular response to hypoxia (31-33). One limitation of our study was that we did not verify whether DFO induced autophagy through HIF-1 $\alpha$ stabilization.

Studies suggest that autophagy is a double-edged sword, with both beneficial and harmful potential in cancer (34). Autophagy is the cellular pathway that mediates lysosomal degradation of intracellular long-lived macromolecules or organelles for subsequent reuse under starvation, or stress such as oxidative stress, endoplasmic reticulum stress, accumulation of abnormal protein and physiological conditions of differentiation $(35,36)$. Autophagy inhibitor, chloroquine (CQ), is widely used to inhibit the maturation of autophagosomes into degradative autolysosomes (37). We identified that CQ, DFO and TRAIL-treated cells underwent cell death to a greater extend than DFO and TRAIL-treated cells (Fig. 4). We suggest that DFO-induced autophagy is a protective effect against TRAIL in colon cancer cells. CQ-mediated inhibition of autophagy interrupted the protective effect of autophagy.

Many studies suggest that TRAIL induces autophagy in several types of cancer cells $(38,39)$. Yet, our results showed that TRAIL treatment did not mediate autophagy marker, LC3-II (Fig. 3A). Rather, TRAIL-treated LC3-II transformation was decreased when compared with the control. Thus, we suggest that TRAIL is not associated with autophagy in HCT116 colon cancer cells. Ikeda et al proposed that DFO promoted Akt activation (40). As shown in Fig. 2A and C, DFO treatment activated Akt phosphorylation dose-dependently. 

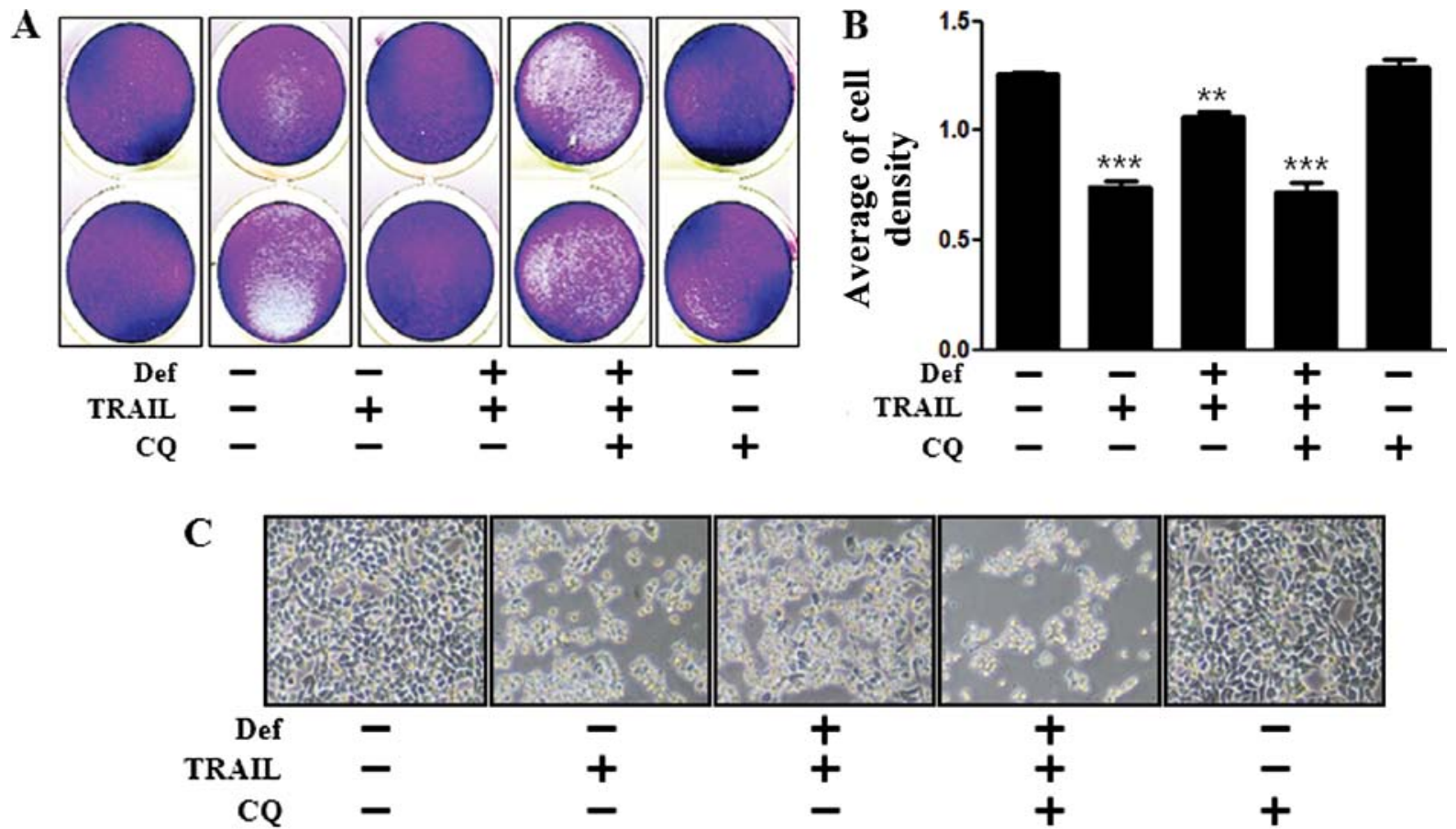

D

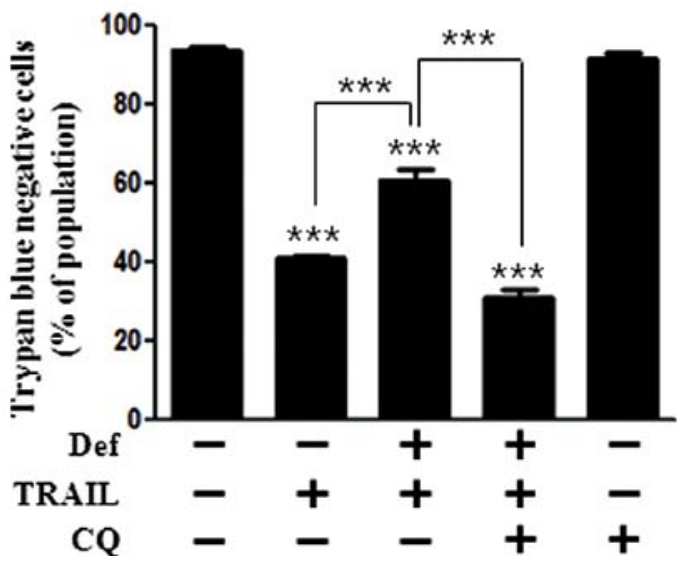

Figure 4. Deferoxamine inhibits TRAIL-induced apoptosis through autophagy. (A) HCT116 colon cancer cells were pretreated with autophagy inhibitor (chloroquine; CQ) $(1 \mathrm{~h}$ ) and then exposed to deferoxamine (Def) $(200 \mu \mathrm{M})$ for $24 \mathrm{~h}$ and TRAIL for $6 \mathrm{~h}$. (B) The bar graph indicates the averages of the density of cells dyed with crystal violet. (C) Cell viability was measured by images captured with a light microscope (magnification, x100), and (D) trypan blue dye exclusion assay. ${ }^{*} \mathrm{p}<0.05,{ }^{* *} \mathrm{p}<0.01,{ }^{* * *} \mathrm{p}<0.001$; significant differences between the control and each treatment group. TRAIL, tumor necrosis factor (TNF)related apoptosis-inducing ligand.

These findings support our suggestion that DFO protects against TRAIL-induced cell death through survival signaling in colon cancer cells.

The crosstalk between autophagy and apoptosis is intricate and sometimes contradictory; however, it is an important determinant of the overall fate of the cell. This report is the first to indicate that DFO-mediated autophagy may play a critical role in cell protection against TRAIL-induced cytotoxicity in colon cancer.

The purpose of the present study was to investigate the role of DFO in TRAIL-induced cell death and the possible mechanism in human colon cancer cells. The results indicated that DFO-induced autophagy flux inhibited the TRAIL-mediated anticancer effect and also suggest that DFO can be a suppressor of anticancer therapy, particularly in TRAIL-mediated colorectal cancer therapy.

\section{Acknowledgements}

This study was supported by a grant from the National Research Foundation of Korea (NRF), funded by the Korean Government (2013R1A1A2063931).

\section{References}

1. Steller H: Mechanisms and genes of cellular suicide. Science 267: 1445-1449, 1995.

2. Hickman JA: Apoptosis induced by anticancer drugs. Cancer Metastasis Rev 11: 121-139, 1992.

3. Zhang JY: Apoptosis-based anticancer drugs. Nat Rev Drug Discov 1: 101-102, 2002.

4. MacFarlane M: TRAIL-induced signalling and apoptosis. Toxicol Lett 139: 89-97, 2003.

5. Lieu PT, Heiskala M, Peterson PA and Yang Y: The roles of iron in health and disease. Mol Aspects Med 22: 1-87, 2001. 
6. Toyokuni S: Iron-induced carcinogenesis: the role of redox regulation. Free Radic Biol Med 20: 553-566, 1996.

7. Nelson RL: Dietary iron and colorectal cancer risk. Free Radic Biol Med 12: 161-168, 1992.

8. Bedford MR, Ford SJ, Horniblow RD, Iqbal TH and Tselepis C: Iron chelation in the treatment of cancer: a new role for deferasirox? J Clin Pharmacol 53: 885-891, 2013

9. Nurtjahja-Tjendraputra E, Fu D, Phang JM and Richardson DR: Iron chelation regulates cyclin D1 expression via the proteasome: a link to iron deficiency-mediated growth suppression. Blood 109: 4045-4054, 2007.

10. Fu D and Richardson DR: Iron chelation and regulation of the cell cycle: 2 mechanisms of posttranscriptional regulation of the universal cyclin-dependent kinase inhibitor $\mathrm{p} 21^{\mathrm{CIP1} / \mathrm{WAFl}}$ by iron depletion. Blood 110: 752-761, 2007.

11. Hershko C: Control of disease by selective iron depletion: a novel therapeutic strategy utilizing iron chelators. Baillieres Clin Haematol 7: 965-1000, 1994.

12. Olivieri NF and Brittenham GM: Iron-chelating therapy and the treatment of thalassemia. Blood 89: 739-761, 1997.

13. Yun Z, Maecker HL, Johnson RS and Giaccia AJ: Inhibition of $P P A R \gamma 2$ gene expression by the HIF-1-regulated gene DEC1/Stra13: a mechanism for regulation of adipogenesis by hypoxia. Dev Cell 2: 331-341, 2002.

14. Nakayama K: Cellular signal transduction of the hypoxia response. J Biochem 146: 757-765, 2009.

15. Semenza GL: Targeting HIF-1 for cancer therapy. Nat Rev Cancer 3: 721-732, 2003

16. Deter RL and De Duve C: Influence of glucagon, an inducer of cellular autophagy, on some physical properties of rat liver lysosomes. J Cell Biol 33: 437-449, 1967.

17. Levine B and Kroemer G: Autophagy in the pathogenesis of disease. Cell 132: 27-42, 2008

18. Boya P, González-Polo RA, Casares N, et al: Inhibition of macroautophagy triggers apoptosis. Mol Cell Biol 25: 1025-1040, 2005

19. Karantza-Wadsworth V, Patel S, Kravchuk O, et al: Autophagy mitigates metabolic stress and genome damage in mammary tumorigenesis. Genes Dev 21: 1621-1635, 2007.

20. Degenhardt K, Mathew R, Beaudoin B, et al: Autophagy promotes tumor cell survival and restricts necrosis, inflammation, and tumorigenesis. Cancer Cell 10: 51-64, 2006.

21. Lum JJ, Bauer DE, Kong M, et al: Growth factor regulation of autophagy and cell survival in the absence of apoptosis. Cell 120 : 237-248, 2005.

22. Hu YL, DeLay M, Jahangiri A, et al: Hypoxia-induced autophagy promotes tumor cell survival and adaptation to antiangiogenic treatment in glioblastoma. Cancer Res 72: 1773-1783, 2012.

23. Chaudhari AA, Seol JW, Lee YJ, Seol DW and Park SY: Hypoxia protects articular chondrocytes from thapsigargin-induced apoptosis. Biochem Biophys Res Commun 381: 513-517, 2009.
24. Agarwal E, Brattain MG and Chowdhury S: Cell survival and metastasis regulation by Akt signaling in colorectal cancer. Cell Signal 25: 1711-1719, 2013.

25. Degterev A, Boyce M and Yuan J: A decade of caspases. Oncogene 22: 8543-8567, 2003.

26. Rubinsztein DC, Cuervo AM, Ravikumar B, et al: In search of an 'autophagomometer'. Autophagy 5: 585-589, 2009.

27. Matsumoto G, Wada K, Okuno M, Kurosawa M and Nukina N: Serine 403 phosphorylation of p62/SQSTM1 regulates selective autophagic clearance of ubiquitinated proteins. Mol Cell 44: 279-289, 2011

28. Kroemer G and Jäättelä M: Lysosomes and autophagy in cell death control. Nat Rev Cancer 5: 886-897, 2005.

29. Liu Y, Cui Y, Shi M,Zhang Q, Wang Q and Chen X: Deferoxamine promotes MDA-MB-231 cell migration and invasion through increased ROS-dependent HIF-1 $\alpha$ accumulation. Cell Physiol Biochem 33: 1036-1046, 2014.

30. Bertout JA, Patel SA and Simon MC: The impact of $\mathrm{O}_{2}$ availability on human cancer. Nat Rev Cancer 8: 967-975, 2008.

31. Mazure NM and Pouysségur J: Atypical BH3-domains of BNIP3 and BNIP3L lead to autophagy in hypoxia. Autophagy 5: 868-869, 2009.

32. Gustafsson AB and Gottlieb RA: Autophagy in ischemic heart disease. Circ Res 104: 150-158, 2009.

33. Wang K,Liu R, Li J, et al: Quercetin induces protective autophagy in gastric cancer cells: involvement of Akt-mTOR- and hypoxiainduced factor $1 \alpha$-mediated signaling. Autophagy 7: 966-978, 2011.

34. White E and DiPaola RS: The double-edged sword of autophagy modulation in cancer. Clin Cancer Res 15: 5308-5316, 2009.

35. Glick D, Barth S and Macleod KF: Autophagy: cellular and molecular mechanisms. J Pathol 221: 3-12, 2010.

36. Mizushima N: Autophagy: process and function. Genes Dev 21: 2861-2873, 2007.

37. Mizushima N, Yoshimori $\mathrm{T}$ and Levine B: Methods in mammalian autophagy research. Cell 140: 313-326, 2010.

38. Singh K, Sharma A, Mir MC, et al: Autophagic flux determines cell death and survival in response to Apo2L/TRAIL (dulanermin). Mol Cancer 13: 70, 2014.

39. Park KJ, Lee SH, Kim TI, et al: A human scFv antibody against TRAIL receptor 2 induces autophagic cell death in both TRAILsensitive and TRAIL-resistant cancer cells. Cancer Res 67: 7327-7334, 2007.

40. Ikeda Y, Tajima S, Yoshida S, et al: Deferoxamine promotes angiogenesis via the activation of vascular endothelial cell function. Atherosclerosis 215: 339-347, 2011. 